Remarques sur « modes » et « modalités » dans les grammaires générales de l'époque classique (France / Angleterre)

André Joly

\title{
CpenEdition
}

Journals

Édition électronique

URL : https://journals.openedition.org/ml/168

DOI : $10.4000 / \mathrm{ml} .168$

ISSN : 2274-0511

Éditeur

Association Modèles linguistiques

Édition imprimée

Date de publication : 1 janvier 2011

Pagination : 19-30

Référence électronique

André Joly, "Remarques sur « modes » et « modalités » dans les grammaires générales de l'époque classique (France / Angleterre) », Modèles linguistiques [En ligne], 63 | 2011, mis en ligne le 28 décembre 2012, consulté le 01 juillet 2021. URL : http://journals.openedition.org/ml/168 ; DOI : https://doi.org/10.4000/ml.168 


\section{Remarques sur " modes " et " modalités » dans les grammaires générales de l'époque classique (France / Angleterre)}

André Joly

\section{Un siècle et demi de grammaire générale}

Le présent essai a été conçu comme un petit guide pour la lecture de l'anthologie qu'on trouvera, ci-après, sous le titre Recueil de textes des XVII et XVIII' siècles.

Je prends comme terminus a quo de l' « époque classique »1660, date de publication de la Grammaire générale et raisonnée d'Arnaud et Lancelot, dite Grammaire de Port-Royal ; comme terminus ad quem, 1796, année où paraît la traduction française de Hermès, ou recherches philosophiques sur la grammaire universelle de James Harris (1751). Ces limites sont évidemment théoriques. Mais le point de vue adopté ici est que la grammaire générale, qui continuera encore un peu sur sa lancée au XIXe siècle, surtout sous la forme de rééditions d'auteurs devenus classiques (Dumarsais, Beauzée, Condillac), a atteint son apogée en France à la fin du XVIII e avec la traduction de Hermès par François Thurot. Accompagnée de nombreuses remarques et d'un Discours préliminaire que l'on peut considérer comme la première histoire de la grammaire ${ }^{1}$.

On sait que le XVIII est le grand siècle de la grammaire " générale ", prélude à ce qui deviendra la « linguistique générale » par rapport aux grammaires « particulières ». En 1730, Restaut, explique pourquoi il faut distinguer ces deux types de grammaire :

[1] Il y a dans chaque langue deux espèces de principes. Les uns sont généraux et communs à toutes les langues, parce qu'ils sont pris dans la nature même des choses, et dans les différentes opérations dont l'esprit de l'homme est capable : tels que sont les définitions et l'usage des noms, des verbes, et de plupart des autres parties du discours. Les autres principes sont ceux qui ne regardent que les mots ou la manière de s'exprimer, et qui sont propres à chaque langue en particulier » (Préface à Principes généraux et raisonnés de la grammaire française, p. xiii).

1. Voir, Thurot, 1970, dans les Références bibliographiques. 
La grammaire générale, appelée aussi raisonnée, universelle, ou philosophique, se situe parfois à un niveau de rationalisation apparemment sans prise sur la "réalité » linguistique. Certes, les " grammairiensphilosophes », comme on les appelle parfois, sont avant tout des théoriciens qui pourraient paraître peu soucieux des données et des contraintes empiriques. Or rien n'est plus inexact.

Tous ces grammairiens-philosophes, de Port-Royal aux Idéologues, ont des préoccupations pédagogiques et ils s'emploient à trouver les applications possibles de leur réflexions sur le langage. Au moins au début de leur carrière, ce sont tous des enseignants. Ainsi Claude Lancelot (1615-1695), disciple de Saint-Cyran et co-auteur avec Antoine Arnauld de la Grammaire générale et raisonnée, est un des piliers des "Petites-Écoles » de Port-Royal. Il est aussi l'auteur de célèbres « méthodes » pour « apprendre facilement » le latin, le grec, l'italien et l'espagnol, qui renouvellent la pédagogie en introduisant la langue maternelle comme métalangue dans l'apprentissage d'une langue étrangère. C'était introduire l'analyse dans l'étude du langage.

Parmi les grammairiens-philosophes les plus connus, Dumarsais et Condillac sont eux-mêmes auteurs de manuels d'apprentissage du français. Ils sont tous engagés, à des titres divers, dans des tentatives de réformes pédagogiques ${ }^{2}$. Locke, dont l'influence sur Condillac est bien connue, avait donné le ton en exposant ses idées sur la manière de former un gentleman dans Thoughts on Education (1693). Traduit en français dès 1695, l'ouvrage avait été reçu avec enthousiasme. A l'extrême fin de l'époque classique, Thurot, le traducteur de Harris, rend compte de la méthode de Pestalozzi (1746-1827), disciple de Rousseau, dans un article intitulé Apologie de Socrate (1806) ${ }^{3}$. Il y met en valeur le rôle de l'association et l'importance du concept d'aperception en pédagogie. Tout cela est, bien entendu, étroitement lié à l'intérêt des philosophes des Lumières pour l'enchaînement des idées, de Locke à Destutt de Tracy, en passant par Condillac ${ }^{4}$.

Selon Thurot, « la première bonne grammaire générale fut précédée de bonnes grammaires particulières ». Par ailleurs, " la meilleure logique est celle qui suit de près la meilleure grammaire ». La chaîne des causations est donc claire : une bonne description grammaticale conduit à une bonne

2. Cf. Malmberg (1994), pp. 233-234 et Thurot (1970) ; sur l'influence de Komensky, alias Comenius, v. Chevalier (1968), pp. 74, 78, 383-386, 619.

3. Sur Pestalozzi (1746-1827) disciple de Rousseau, v. Malmberg, p. 252.

4. Sur Condillac, v. Joly (1982). 
logique. À Port-Royal, les diverses «méthodes » de Lancelot ont précédé la Grammaire (1660) qui, à son tour, précède la Logique (1662).

De là l'importance de la familiarité avec les faits concrets de la langue de communication. La pédagogie de l'époque essaie toujours et encore de se libérer de l'emprise du latin et de son mode de pensée. On n'insistera jamais assez sur le fait qu'à l'époque classique, l'analyse du langage n'est pas que théorique, contrairement à une idée trop répandue. En réalité, elle est fondée sur l'observation des faits empiriques et orientée vers les applications pédagogiques. Du reste, les grammaires scolaires s'en inspirent souvent, en en donnant des « digests » ; ils vont même jusqu'au plagiat. Ce sera particulièrement le cas au XIXe siècle ${ }^{5}$.

Pourquoi avoir réduit mon enquête à la France et à l'Angleterre ? C'est que, du point de vue de la réflexion sur le langage, si le XVle siècle est " espagnol » avec la Minerve de Sanctius (1587) ${ }^{6}$, dont l'influence a été considérable en Europe ; si le XIX est avant tout " allemand », avec la linguistique comparative, puis historique, les XVII et XVIII siècles, voués à la grammaire générale, sont essentiellement français et anglais. La " grammaire générale » en France correspond à la " grammaire universelle » au Royaume-Uni. À l'époque, les rapports entre les deux pays sont étroits dans de nombreux domaines, notamment politique, littéraire et philosophique, bien qu'ils soient très souvent antagoniques, en particulier pour ce qui est de l'étude du langage.

Ainsi, la Grammaire générale et raisonnée d'Arnauld et Lancelot, ne fait apparemment pas fortune en Angleterre, puisqu'elle ne sera traduite qu'en 1753, soit près d'un siècle après sa parution en France. Elle est cependant plagiée, y compris dans des grammaires élémentaires, comme par exemple A Grammar of the English Tongue (1711), dite de Brightland, dont les abondantes notes sont une paraphrase de Port-Royal et, la même année, Essay Towards a Practical English Grammar de James Greenwood. Mais plus tard, au milieu du siècle (1751), l'influence de Port-Royal sur James Harris est nulle. Harris ne la mentionne que deux fois, et de manière plutôt désinvolte. Arnauld et Lancelot sont vus comme des « able writers » (que Thurot surtraduit par «très habiles ») et leur ouvrage est présenté comme un "ingenious French Treatise », traduit par "un excellent ouvrage ». Visiblement, Thurot, en rajoute ou traduit à côté. Les raisons en sont évidentes. En résumé, on peut dire que, si la première moitié du XVIII est, en Angleterre, sous l'influence occulte et diluée de

5. Voir ici même l'article de C. Carlut, A. Joly et C. Noble.

6. Il existe une première version de la Minerve, cf. M. Breva-Claramonte, dans Historiographia Linguistica 2,1, 1975. 
Port-Royal, la seconde moitié, entièrement dominée par le Hermès de Harris, échappe au "cartésianisme » et marque un retour à l'aristotélisme7. La comparaison du traitement du mode et des modalités en France et en Angleterre peut donc réserver des surprises.

\section{Le mystère des modes et des modalités}

Pour commencer, les modes existent-ils et, s'ils existent, combien y en a-til ? Questions préjudicielles qu'on se pose en France, où on les appelle aussi «mœufs », qu'on se pose aussi en Angleterre, où on leur donne les noms de «modes » et de « moods », ce dernier terme tirant dans le sens de « modalité » 8 .

Pour ce qui est de l'existence et de la nature des modes, Anglais et Français vont d'un scepticisme mitigé à la négation pure et simple. Ils passent d'un extrême à l'autre :

[2] [...] la nature des Modes est si peu connue des grammairiens, qu'ils ne s'accordent point sur le nombre de ceux qu'il faut reconnaître dans une langue ; ce qui indique, au gré du grammairien espagnol [Sanctius], que la distinction des Modes est chimérique et uniquement propre à répandre des ténèbres dans la Grammaire (Beauzée, 1767).

[3] It is well observed [...] « that if we will constitute as many moods as there are various modifications wherewith a verb or affirmation may be affected, we must multiply them to a very great number ; and, besides the Indicative, Subjunctive, Potential, Optative, Imperative, and Interrogation, have also a Permissive, an Hortative, a Precative, a Concessive, a Mandative, a mode to express volition, and another to signify duty » - which, instead of improving the grammatical art, would only render it the more confused and difficult, without adding anything to the regularity or significancy of language » (Beattie, 1783).

[4] In English there are no Moods [...] (Greenwood, 1737).

La difficulté centrale à laquelle se heurtent grammairiens anglais et français en matière de mode et de modalité repose sur la question de savoir s'il faut privilégier le sens ou bien la forme. On dira par exemple que le grec a un optatif parce qu'il a une forme spécifique pour exprimer le souhait, forme qui n'existe pas en latin. Donc, selon certains, le latin n'a

7. Voir mon introduction à Hermès 1972.

8. Mood, dans le sens grammatical de "mode" apparaît au milieu du XVle siècle. C'est un croisement sémantique avec l'autre sens de mood, plus ancien, lui, puisqu'il est d'origine germanique : mōd en vieil anglais (cf. all. Mut) signifie, entre autres, "état d'esprit". Il était donc aisé de glisser du sens de "mode grammatical" à celui de "modalité", "affection de l'âme", comme on disait dans l'Antiquité (v. infra). 
pas d'optatif ; mais d'autres font observer qu'il a la particule utinam, qui en tient lieu. Le souhait s'exprimerait alors de manière périphrastique.

Par ailleurs, alors que le français a toute une série d'inflexions pour le subjonctif, l'anglais n'en a pas, la seule opposition entre l' « indicatif » et le "subjonctif » étant la troisième personne, qui n'est pas marquée au subjonctif (he loves vs that he love). C'est la raison pour laquelle, selon de nombreux grammairiens, l'anglais n'aurait pas de mode subjonctif. Harris en explique les conséquences, ici dans la traduction de Thurot:

[5] Les langues modernes, qui ont encore bien moins [que le latin] de ces sortes de variations, ont toutes été dans la nécessité d'admettre deux auxiliaires au moins, c'est-à-dire, ceux qui dans chaque langue signifient être ou avoir. Quant à la langue anglaise, elle est si pauvre à cet égard, qu'elle n'a point de variations pour les modes, et qu'elle n'en a qu'une seule pour le temps ; c'est l'aoriste du passé. Ainsi de write [écrire], nous faisons I wrote [j'écrivis], etc. De là vient que pour exprimer les différences des temps et des modes, nous sommes obligés d'employer jusqu'à sept verbes auxiliaires (Hermes [1751] 1796, I, VIII, p.138 note).

En ce point, Thurot a cru bon de supprimer un passage de l'original. Harris y donne en note la liste des auxiliaires en question, que je reproduis telle quelle : Do, Am, Have, Shall, Will, May, Can. A quoi il faut ajouter, au vu des exemples qu'il donne, les formes de prétérit : did, was, had, should, would, might, could, soit, au total, 14 formes. Harris commente :

[6] [Auxiliars] which we use sometimes singly, as when we say, I am writing, I have written ; sometimes two together, as, I have been writing, I should have written; sometimes no less than three, as I might have been lost, he could have been preserved.

Et il termine ainsi cette longue note :

[7] But these peculiarities are perhaps foreign to our design, which is rather to inquire concerning Grammar Universal.

Cette phrase est remplacée dans la $3^{e}$ édition de Hermes (1771) par un renvoi à la grammaire de Lowth, qui est une grammaire « particulière » de l'anglais (1762, 2e éd. 1763). Où l'on voit quelle est l'« utilité » de la distinction entre grammaire générale et grammaire particulière.

En France, avec Beauzée, on quitte le domaine de la forme pour entrer dans celui du sens :

[8] Les Modes ont reçu le nom général de Modes, non par rapport à la différence des terminaisons, mais relativement à la différence des aspects sous lesquels on envisage la signification fondamentale du verbe (1767, Liv ; III, chap. VI, p. 341; souligné par moi). Avec cette précision : «Les Modes ne se rapportent pas seulement à la Syntaxe : il n'y a que le subjonctif qui ait rapport à cet objet; les autres modes sont simplement différentes manières d'envisager la signification fondamentale $d u$ verbe». 
Les commentaires de Harris et de Beauzée, qui comptent parmi les grammairiens-philosophes les plus célèbres, illustrent deux points de vue diamétralement opposés quant à l'identification des modes et, par implication, des modalités :

(1) le point de vue de Harris est strictement formel : on s'attache au signifiant, à savoir les «terminaisons », ou les auxiliaires qui en tiennent lieu.

(2) le point de vue sémantico-énonciatif (Beauzée) où l'on est invité à se concentrer sur le signifié, en essayant d'identifier les « aspects » " sous lesquels est considérée la signification du verbe ».

Le point de vue de Beauzée sera, près de deux siècles plus tard,celui de Damourette et Pichon :

[9] [...] au point de vue psychologique, l'esprit d'un Français aperçoit à peine, lors de l'emploi même, la similitude matérielle entre les formes du savez [lire : indicatif] et celles du sachiez [lire : subjonctif]. Soit par exemple une phrase comme :

Mais j'aime mieux que tu ne parles pas de ma fille. Non!

(Duhamel, Le Club des Lyonnais, p. 183)

Ni celui qui la profère, ni celui qui l'entend ne songe à y comprendre parles comme un indicatif.

\section{Chiffres et statistiques}

Pour une période d'environ deux cents ans (entre la fin du XVle et la fin du XVIIIe), Ian Michael (1970) a pu recenser 258 grammaires anglaises, scolaires et savantes. Pour ce qui est du traitement du mode, les chiffres suivants ont été relevés :

- 35 d'entre elles ignorent purement et simplement l'existence d'un quelconque mode, soit $13,5 \%$,

- 19 déclarent explicitement que l'anglais n'a pas de modes, soit environ $7 \%$

Ce qui signifie qu'une grammaire sur cinq ignore ou refuse l'existence des modes en anglais (cf. ci-dessus le point de vue de Greenwood 1737, cit. [4]).

Toutefois les 204 grammaires qui admettent l'existence des modes sont loin d'être d'accord, à la fois sur le nombre de ces modes et sur la manière de les classer. On relève en effet 22 types de classements différents. De plus, à l'intérieur de ces classements, le nombre des modes identifiés varie de 2 à $10 .$. 
On aurait pu s'attendre à ce que le système à 5 modes, hérité de la tradition gréco-latine, que l'on doit à Denys le Thrace (170-90 av J.-C), viendrait en tête, à savoir, dans l'ordre : indicatif, impératif, optatif, subjonctif, infinitif. Or tel n'est pas le cas, du moins en Angleterre, où le classement à 4 modes l'emporte largement : 108 grammaires l'adoptent, soit près de $42 \%$.

Mais là cesse l'accord : les 4 modes retenus varient d'une grammaire à l'autre. Le système qui a la faveur de la majorité retient, dans l'ordre : indicatif, impératif, infinitif, subjonctif. Pourquoi cet ordre ? Mystère. Il faut toute de même noter un « progrès » par rapport à la tradition grécolatine : dans la majorité des cas, on a laissé tomber l'optatif qui, rappelonsle, n'est morphologiquement marqué qu'en grec. L'optatif a cependant la vie dure, puisqu'on le retrouve encore dans une grammaire à l'extrême fin du XVIIII siècle...

En Angleterre, le système de classement à 5 modes arrive en seconde position, mais bien après le classement à 4 modes : 51 grammaires, soit seulement près de $20 \%$, l'adoptent. Je laisse de côté les autres classements à $n$ modes, qui concernent le tiers des grammaires examinées.

Une dernière statistique qui, cette fois, concerne l'ensemble des 22 types de classement. Le mode qui vient en tête de façon absolue est l'indicatif (22/22). Il est suivi par l'impératif (19/22), puis, en troisième position, à égalité, par l'infinitif et le subjonctif (15/22).

$\mathrm{Au}$ terme de ce parcours labyrinthique entre chiffres et statistiques, qui a au moins le mérite de mettre les choses en perspective, le lecteur le plus optimiste pourrait penser y voir un peu plus clair, mais à quel prix ? En laissant au second, sinon au troisième plan, dans cette jungle de 250 grammaires, plus de la moitié des systèmes de classement. La « clarté » est donc très relative. Viendra-t-elle de l'examen des grammaires françaises ?

On serait tenté de le croire, compte tenu du fait que mon corpus en français est beaucoup plus modeste. Je n'ai en effet examiné qu'une cinquantaine de grammaires et n'en ai cité qu'une vingtaine dans l'anthologie qu'on trouvera à la suite du présent article. L'avantage est que l'ensemble est beaucoup plus homogène, la plus grande partie de ces ouvrages ayant des visées théoriques. Parmi eux, j'en ai retenu une petite dizaine parmi les plus connus ou les plus souvent cités, à l'exclusion de Vaugelas, chez qui il n'est question que de l'opposition indicatif/ subjonctif.

Sur ce choix volontairement restreint, on aurait pu s'attendre à une sorte de consensus. Il n'en est rien. Les auteurs finalement retenus (v. 
Anthologie 1) proposent quatre systèmes différents, dans l'ordre indiqué cidessous :

- à 2 modes : indicatif et subjonctif (Dangeau).

- à 4 modes : indicatif, impératif, subjonctif, infinitif (Restaut, Dumarsais, Condillac).

- à 5 modes : indicatif, subjonctif, optatif, concessif, impératif (PortRoyal) ; indicatif, impératif, optatif, subjonctif, infinitif , (Furetière).

- à 6 modes : infinitif, gérondif, participe, indicatif, suppositif, subjonctif (Girard) ; indicatif, infinitif, participe, impératif, suppositif, subjonctif (Beauzée).

Il est évident qu'on n'y voit pas beaucoup plus clair que dans les grammaires anglaises.

\section{Deux questions et quelques éléments de réponse}

On peut se poser ici deux grandes questions étroitement liées :

$1^{\circ}$ Pourquoi cette catégorie grammaticale a-t-elle tant divisé les grammairiens, peut-être plus que toute autre ? Je n'apporte aucune réponse.

$2^{\circ}$ Pourquoi, sauf erreur, aucun grammairien de l'époque n'a cherché à justifier sérieusement l'ordre de présentation de ces "modes", alors que c'est un sujet de controverses dans l'Antiquité ? Cette question est peut-être la plus importante, car de l'ordre choisi - s'il n'est pas aléatoire - dépend l'idée que l'on se fait du système d'ensemble.

La tradition remontant à l'Antiquité offrait une conception articulée sur deux idées : (a) que le mode est l'expression d'une " affection de l'âme ", (b) que ladite expression passe essentiellement par le verbe. Apollonius Dyscole (grammairien grec d'Alexandrie, 2e s. ap. J.-C.) est le premier qui expose clairement cette conception :

[10] Les verbes sont spécialement destinés à exprimer la disposition de l'âme (psuchikè diathesis) (De Synt., 1. III, c. 13).

On remarquera qu'Apollonius précise « spécialement » (éksairétôs), ce qui donne à entendre que d'autres éléments peuvent aussi servir à exprimer les affections de l'âme. Mais il n'en dit pas davantage.

Des siècles plus tard, Théodore de Gaza (vers 1400-1475), auteur d'une célèbre grammaire grecque publiée à Venise en 1495, puis traduite en latin par Érasme, reprend cette idée, mais sans préciser que c'est le verbe qui exprime spécialement le mode : 
[11] [Le mode est ] une volonté ou une affection de l'âme exprimée par quelque voix ou son articulé (Gramm. 1. IV) [bélèma én pathèma psuchès].

On a sans doute là l'origine de la tradition psychologisante liée à la notion de mode. Elle est centrée sur le sujet parlant, sur sa subjectivité et son affectivité. Mais, comme on l'a vu, on confondra longtemps le mode inscrit dans la conjugaison du verbe et le mode correspondant au type d'énoncé (assertif, interrogatif, etc.). La plupart des grammaires modernes souffrent toujours de cette confusion.

Selon une tradition qui, on l'a vu, remonte à Apollonius Dyscole, la détermination du mode est étroitement liée à la conception qu'on a du verbe. À l'époque classique, et dans la tradition antique, on peut distinguer quatre conceptions $d u$ verbe qui peuvent se situer sur un continuum : (a) logique, (b) formelle, (c) sémantico-formelle, (d) sémantique :

(a) Conception logique:

[12] Un mot dont le principal usage est de signifier l'affirmation [et] de marquer la liaison que nous faisons dans notre esprit des deux termes d'une proposition (Grammaire de Port-Royal, 1660).

Cette conception s'inscrit dans la tradition aristotélicienne. En effet, selon Aristote (IVe s. av. J.-C) :

[13] Le verbe [rêma] est ce qui ajoute à sa propre signification celle du temps [...] et il indique toujours quelque chose d'affirmé de quelque autre chose (De l'interprétation, 3).

On notera à ce propos le contresens attaché parfois à la mauvaise compréhension du verbe composé qu'utilise Aristote : "prosémainei ", bien traduit par une périphrase dans la citation ci-dessus. Pro-indique en effet qu'il s'agit d'une « signification » qui vient « s'ajouter » à la signification du verbe. Tout aussi avantageusement, on pourrait dire « consignifie » pour rendre le préverbe pro-. C'est ce qu'ont fait certains grammairiens à l'époque classique. Mais Michel Foucault, à la suite de quelques autres, a fait le contresens que je viens de signaler : contrairement à ce qu'il affirme, le verbe ne « signifie » pas le temps, il le «consignifie ». C'est bien ce que dit Aristote. Nuance des plus importantes sur le plan théorique pour l'analyse du verbe (pour plus de précisions, v. Joly 2008).

Par ailleurs, à la suite d'Aristote, l'idée que le verbe signifie «l'affirmation » traverse toute l'histoire de la grammaire occidentale. C'est une manière pour le moins maladroite de dire que le verbe est porteur du prédicat, de ce qui se dit de quelque chose d'autre, à savoir le «sujet » (subjectum), ou support (sup-positum) de l'énoncé (le « suppost» chez certains 
gramairiens de l'époque classique). "Signifier l'affirmation » est une manière ambiguë de dire que le verbe fait ce que Port-Royal appelle la « liaison... des deux termes d'une proposition » (cit [12]). Or la liaison se fait, comme le précise bien Aristote (cit. [13]), dans le temps. $L^{\prime}$ « affirmation » $n^{\prime}$ est donc pas autre chose que la mise en rapport temporelle d'un apport de signification (prédicat) à un support de signification (sujet). On a là la théorie des rangs de Jespersen, l'incidence de Guillaume, la connexion de Tesnière.

(b) Conception formelle :

$C^{\prime}$ est la tradition qui remonte à Ramus au XVIe siècle :

[14] Verbum est vox numeri cum tempore et persona.

Tradition bien représentée en Angleterre, par exemple par Merriman (1750) qui reproduit textuellement la définition de Ramus :

[15] (The verb is) a word of number, which has both Time and Person.

On la trouve aussi dans une autre formulation de Port-Royal :

[16] (Le verbe est) un mot qui marque l'affirmation de quelque attribut, avec désignation de la personne, du nombre et du temps.

(c) Conception sémantico-formelle:

Cette approche peut être attribuée à Priscien (VIe siècle) :

[17] Verbum est pars orationis cum temporibus et modis, sine casu, agiendi, vel patiendi significativum (le verbe est une partie du discours avec temps et modes, dénuée de cas et signifiant agent ou patient).

Ici, ce sont donc à la fois le sens du verbe et ses «accidents », modaux et temporels, qui sont pris en considération. James White (1761), par exemple, en donne une variante plus développée :

[18] A verb is a word whereby something or other is represented as existing, possessing, acting or being acted upon // at some particular time, past, present or future, and this in various manners.

(d) Conception sémantique :

Seul le sens est retenu. Deux exemples aux deux extrémités du XVIII siècle :

[19] A verb is a word that betokens being, doing, or suffering [...] (James Greenwood, 1711).

[20] Qu'est-ce qu'un verbe de mode indicatif ? - C'est un verbe qui indique l'existence, la possession, ou l'action, et fait un sens complet sans dépendre d'un autre verbe qui le précède » (Gaultier, 1787).

La dernière citation montre avec quelle aisance on peut passer de la définition du verbe au mode. Le verbe, " âme de la phrase ", moteur de la prédication, avec son sens et ses « accidents », est en quelque sorte 
l'antichambre du mode, dont l'expression, c'est bien connu, précède historiquement celle du temps.

ERIS, Babel, EA 2649

\section{RÉFÉRENCES}

ARNAULD, Antoine \& LANCELOT, Claude ([1660] 1966), Grammaire générale et raisonnée, ou La Grammaire de Port-Royal, édition critique présentée par Herbert E. Brekle, F. Frommann, Stuttgart-Bad Cannstatt.

BARATIN, Marc \& DESBORDES, Françoise (1981), L'analyse linguistique dans l'antiquité classique, Klincksieck, Paris.

CHEVALIER, Jean-Claude (1968), Histoire de la syntaxe. Naissance de la notion de complément dans la grammaire française (1530-1750), Droz, Genève.

HARRIS, James (1972), Hermès, ou recherches philosophiques sur la grammaire universelle de James Harris (1751), traduction de François Thurot, édition, introduction et notes, Droz, Genève-Paris.

JOLY, André (1970), v. Thurot.

JOLY, André (1976), « Le débat sur les parties du discours à l'époque classique ", Zeitschrift für Phonetik, Sprachwissenschaft und Kommunikationsforschung, 5/6, Band 29.

JOLY, André (1982) " De la théorie du langage à l'analyse d'une langue », dans Condillac et les problèmes du langage, Slatkine, Genève.

JOLY, André (1986), « Temps et verbe dans les grammaires anglaises de l'époque classique » Histoire, Épistémologie, Langage, vol. 7.

JOLY, André (2008) « Notes de lectures : de quelques malentendus sur la définition du verbe ", Modèles linguistiques, vol. 57.

MALMBERG, Bertil (1991), Histoire de la linguistique, de Sumer à Saussure, PUF, Paris.

MICHAEL, Ian (1970), English Grammatical Categories and the Tradition to 1800, Cambridge University Press.

THUROT, François ([1796] 1970), Tableau des progrès de la science grammaticale, édition, introduction et notes d'André Joly, Bordeaux, Ducros ; $2^{\mathrm{e}}$ édition, L'Harmattan, 2004. 


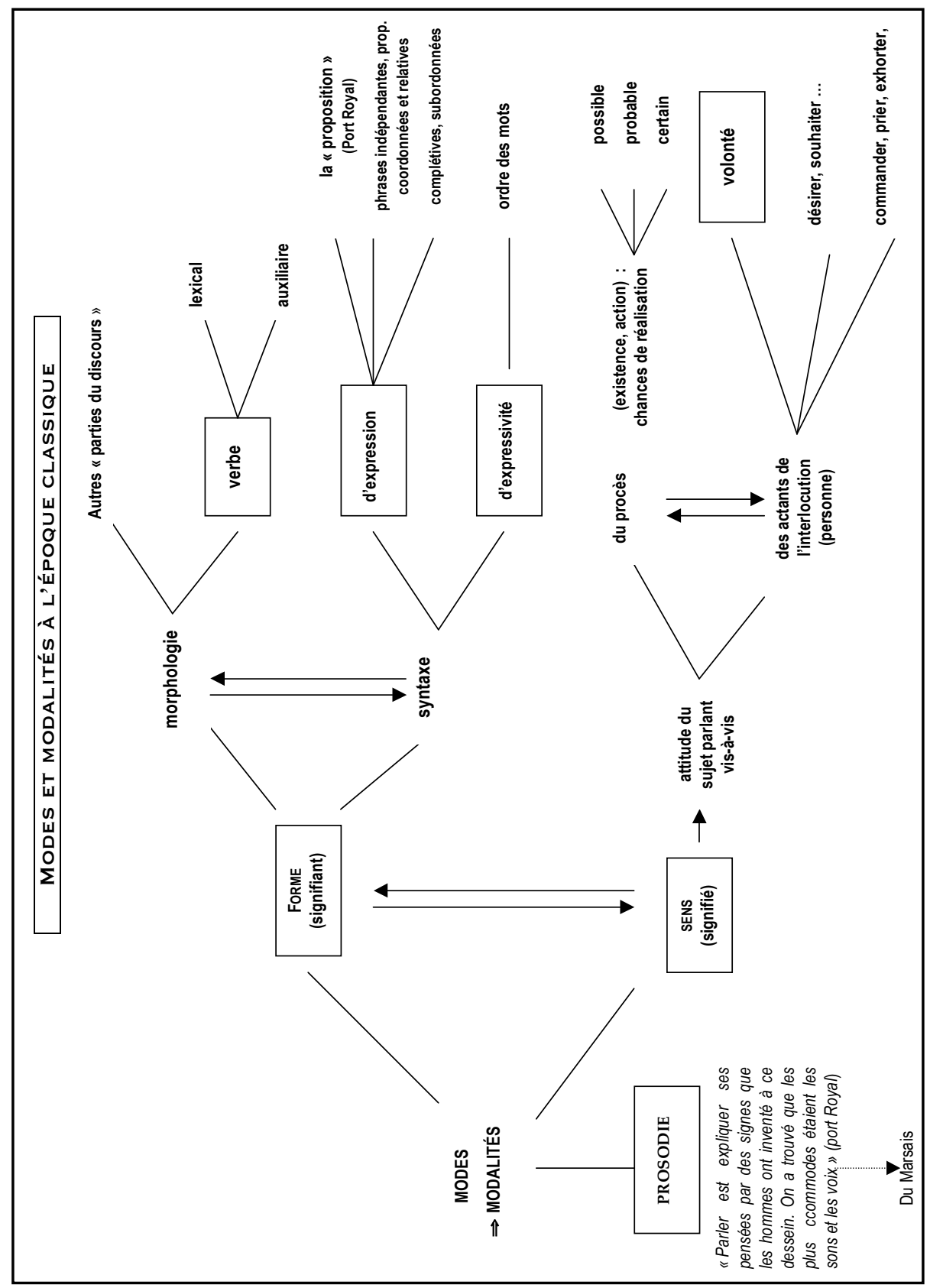

